\title{
On-line adaptive side-by-side human robot companion to approach a moving person to interact
}

\author{
Ely Repiso, Anaís Garrell, and Alberto Sanfeliu \\ Institut de Robòtica i Informàtica Industrial, CSIC-UPC \\ \{erepiso, agarrell, sanfeliu\}@iri.upc.edu
}

\begin{abstract}
In this paper, we present an on-line adaptive side-by-side human-robot companion to approach a moving person to interact with. Our framework makes the pair robot-human capable of overpass, in a joint way, the dynamic and static obstacles of the environment while they reach a moving goal, which is the person who wants to interact with the pair. We have defined a new moving final goal that depends on the environment, the movement of the group and the movement of the interacting person. Moreover, we modified the Extended Social Force model to include this new moving goal. The method has been validated over several situations in simulation. This work is an extension of [17].
\end{abstract}

Keywords: Human-Robot companion side-by-side, Robot Navigation, Human-Robot Interaction, Human-Robot Collaboration.

\section{Introduction}

In the future, we aim to find social robots living together with humans in urban areas. To achieve that, robots have to develop several new skills, such as understand or predict human intentions, or navigate together with humans.

Furthermore, if robots have to accompany humans they must deal with several situations, such as guiding people in cities [4, 7], in shopping malls [1], following people and learning new objects and places to future interactions [10], or helping aging people as home care robots to live independently in their homes [9].

In urban environments, humans tend to approach to other people to interact with them in different ways. Getting closer known people in streets, or talking to pedestrians if help is needed, for instance, asking directions or taking a photo. Also, if people tend to navigate in groups and if they want to interact with other person they have to re-organize the group to navigate comfortably.

For a human-robot pair, intercepting a moving person to interact with it is a challenging task, on the one hand, the robot has to anticipate the movement of the moving person and intercept it, while has to navigate in a joint way with the person that accompanies. On the other hand, the group has to avoid together static and dynamic obstacles, such as pedestrians in the environment. In addition, other people and obstacles can occlude the detection and tracking of people, thus, the robot has to deal with it. An example of these situations can be seen in Fig. 1, where the robot has to intercept the person which is the 


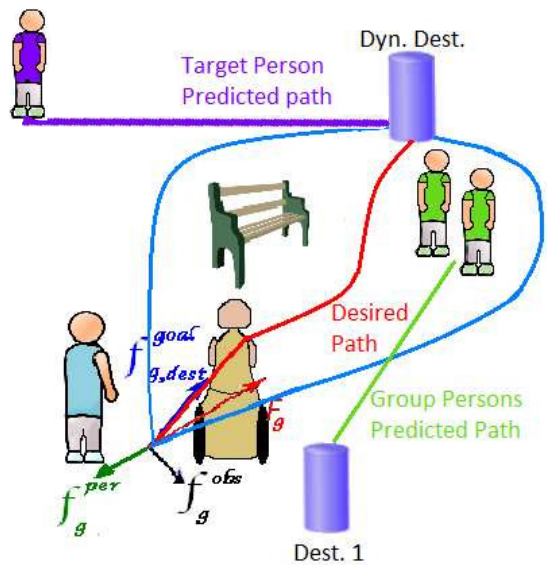

Fig. 1: A couple robot-human moving towards a target person, while one group of people is moving in the opposite direction. Moreover, the robot-human pair has to avoid a bench. We show the three different paths that can be selected in that instance of time. The red path is the best path to go to the moving target person and the blue paths are the discarded paths, due to their highest cost.

moving target goal of the group while has to avoid static and dynamic obstacles. Finally, the robot needs to perform well several complex tasks as anticipation, deal with uncertainties, human-robot interaction with several people, perception, prediction and autonomous navigation.

As a starting point on the companion approaches, some researchers developed reactive approaches for robots to accompany people [16]. Since reactive approaches are not suited to obtain a realistic and safe companion task, some studies included prediction to anticipate the behaviour of the partner and navigate in a more intelligent way. Such as the work presented in [15], where they perform and compare two approaches for walking side-by-side: the leader-follower and the collaborative. In [14], they proposed a framework which describes people walking side-by-side and used this model to predict the future next position of the partner and plan the next robot position. Finally, Burgard et al. [13] explored the possibility to face the problem using reinforcement learning. They apply reinforcement learning to teach a tele-operated robot how to navigate in a cooperative way avoiding collisions with obstacles.

When people interact in near distance, we need to use the proxemic rules [11]. All works agree that the proxemic rules between person and robot are similar to people. Syrdal et al [18] study the near distances between robot and person in different interaction situations: verbal, physical and no interaction. One of their founds was that the females allow the robot to be closer than the males, independently of the approach direction. Some studies [2] found that is better for the robot to approach the person from one of the sides, right-handed people prefer to be approached from the right. . 
In this paper, we go a step further from the previous on-line adaptive side-byside human-robot companion in dynamic environments, [17]. We study several situations that appear when a person accompanied by a robot goes to interact with other pedestrian in a dynamic environment. Then, we plan to use the knowledge of these situations to implement a modification in our approach to allow the group to intercept a moving person to interact with it. The new model includes a framework to calculate a moving goal taken into account the movement of the person, the movement of the group and the best path to go through the obstacles (dynamic or static) of the environment. Then, we reformulate the Extended Social Force Model [12] to include the moving goal which is recalculated in each iteration of our algorithm. This new goal is computed taken into account the robot has to adapt its behavior to deal with the changes of the environment and the two people (the companion person and the person which is the goal) trajectory decisions.

In the remainder of the paper, we start by introducing the Extended Social Force Model previously presented. Thus, a summary of the adaptive companion of Repiso et al. [17] is shown in Sec. 2. Then, the implemented approach is shown in Sec. 3, which includes and extension of the Extended Social Force Model by changing the static goal to a moving goal. In Sec. 4, we explain the results of the simulations, and finally, in Sec. 5, we present the conclusions and some possible lines of future work.

\section{The adaptive companion.}

In this section, we present a short review of the previous paper [17]. The adaptive companion task extends the Anticipate Kinodynamic Planning (AKP) of Ferrer et al. [6] by the addition of a new cost function, Eq. 1, which takes into account the cost of walking depending on the person and robot group configuration. This cost function is added to the cost functions defined in the AKP method [6]. This new cost function allows the robot to take into account the companion task when selects the best navigation path for the group.

$$
J_{c}(S)=\eta\left\|\theta(t)-\theta^{0}\right\|^{2},
$$

where $S$ is the state of the robot and all the people, which includes position, velocity and time and additionally orientation for the robot [5]. The $\theta(t)$ is the desired angle between person and robot in each position of the path at time $t, \eta$ is a normalization parameter, taking into account that $\theta(t) \in[0-\pi]$ and we want to obtain a maximum worst cost of 1 on the front and rear positions respect to the companion person, and $\theta^{0}$ is the best companion angle, which is $\pi / 2$ degrees in our case, to allow the walking side-by-side. Fig. 2-left plots the companion cost of Eq. (1).

$$
\theta=\pi-\arcsin \left(\frac{d_{w}}{R_{i}}\right) \text {, for } \theta \in\left(-\frac{\pi}{2}, \frac{\pi}{2}\right]
$$

Where, $d_{w}$ is the shortest distance between the robot center and the direction of movement of the group. Also, a geometric representation of it can be seen in Fig. 2. Where, $d_{o}$ is the distance between the center of the group and the obstacle, 
$d_{p}$ is the person radius and $R_{e}$ is the radius of the circle containing the inflated robot (radius $R_{r}$ ) and person $\left(R_{p}\right)$.
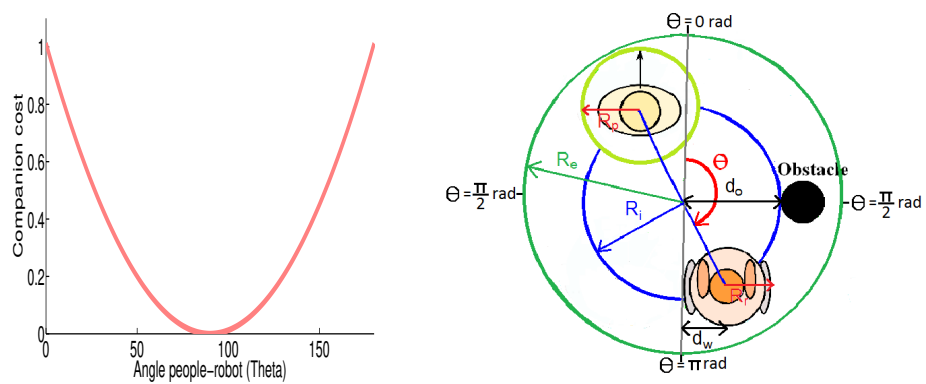

Fig. 2: The right figure shows the cost companion graph and the left figure shows the computation of the companion angle for a possible obstacle collision and the reference of angles that we use to perform the companion task.

Furthermore, we modify the social force model of the AKP to include a force which allows to keep the best companion formation between the person and robot Eq. 3.

$$
\begin{gathered}
\mathbf{F}_{r}=\alpha \mathbf{f}_{r, p}^{\text {goal }}\left(\mathcal{D}_{n}{ }^{p}\right)+\left(\gamma \mathbf{F}_{r}^{p e r}+\delta \mathbf{F}_{r}{ }^{\text {obs }}\right) . \\
1=\alpha+\gamma+\delta
\end{gathered}
$$

where, $\gamma$ and $\delta$ were obtained as described in [3].

The attractive force assuming that the robot $r$ tries to adapt its velocity within a relaxation time $k^{-1}$, but taking into account at the same time the distance and angle of the accompany person to destination $\left(\mathcal{D}_{n}{ }^{p}\right)$, is computed as follows:

$$
\left.\mathbf{f}_{r, p}^{\text {goal }}\left(\mathcal{D}_{n}{ }^{p}\right)=k\left(\mathbf{v}_{r}^{0}\left(\mathcal{D}_{n}{ }^{p}\right)-\mathbf{v}_{r}\right)\right)
$$

Where $\mathbf{v}_{r}^{0}\left(\mathcal{D}_{n}{ }^{p}\right)$ is the desired velocity vector to reach the goal according to the destination $\mathcal{D}_{n}{ }^{p}$ and $\mathbf{v}_{r}$ is the current velocity of the robot. This is the force that keeps the robot in the computed geometrical configuration (robot-human) to allow the joint navigation.

Eq. 14 is applied at each discrete point of the path to reach the goal. Let us consider that there are $t=1,2, \ldots, M$ discrete points in the path, then, the attractive force of the next discrete point $\mathbf{f}_{r, p}^{\text {goal }}{ }^{t+1}$ will be computed as:

$$
\mathbf{f}_{r, p}^{\text {goal }}{ }^{t+1}=k\left(\mathbf{v}_{r}^{0}\left(P_{r}^{t+1}\right)-\mathbf{v}_{r}\left(P_{r}^{t}\right)\right.
$$

Where $P_{r}{ }^{t+1}$ is the next position of the robot taken into account the constraints in distance and angle imposed by the person and $P_{r}{ }^{t}$ is the current position of the robot. Because the robot has always to follow the person and there are constrains in distance and angle between the robot and the person, the computation of the next position of the robot, $P_{r}{ }^{t+1}$, will depend on the next position of the person, $P_{p}{ }^{t+1}$. If we consider the two components of the position of the robot, $P_{r}{ }^{t+1}=\left(X_{r}{ }^{t+1}, Y_{r}{ }^{t+1}\right)$ and of the position of the person, $P_{p}{ }^{t+1}=\left(X_{p}{ }^{t+1}, Y_{p}^{t+1}\right)$, then the computation of $P_{r}{ }^{t+1}$ will be: 


$$
\begin{gathered}
\hat{x}_{r}^{t+1}=\hat{x}_{p}^{t+1}+2 R_{i} \cos \left(\theta_{p}-\operatorname{sgn}\left(\theta_{p}-\theta_{c}\right) \theta\right) \\
\hat{y}_{r}^{t+1}=\hat{y}_{p}^{t+1}+2 R_{i} \sin \left(\theta_{p}-\operatorname{sgn}\left(\theta_{p}-\theta_{c}\right) \theta\right)
\end{gathered}
$$

$2 R_{i}$ is the distance between the robot and the person center positions. $\theta_{p}$ is the person orientation to the destination and is obtained with the Eq. (9), sgn is the sign function. Thus, $\theta_{c}$ is the real companion angle between the person and robot positions. Finally, $\theta$ is the desired companion angle between the robot and the person, Eq. 2, which takes into account the person's position, the orientation of movement and the collisions in each step of each possible path of the group navigation, to obtain more details see [17]. $R_{i}, \theta_{p}, \theta_{c}$ and $\theta$ are computed in the discrete time $t$. These equations make the robot move in one of the sides of the person.

$$
\theta_{p}=\operatorname{atan}\left(\frac{Y_{\text {goal }}-Y_{p}}{X_{\text {goal }}-X_{p}}\right)
$$

where, $\left(X_{\text {goal }}, Y_{\text {goal }}\right)$ is the goal position and $\left(X_{p}, Y_{p}\right)$ is the person position in discrete time $t$.

The repulsive force respect to the other pedestrians in the dynamic environment is:

$$
\mathbf{F}_{r}^{p e r}=\sum_{j \in P} \mathbf{f}_{r, j}^{i n t}
$$

The repulsive force respect to the obstacles in the environment is:

$$
\mathbf{F}_{r}^{o b s}=\sum_{o \in O} \mathbf{f}_{r, o}^{\text {int }}
$$

These repulsive forces are the summation of all the repulsive forces between the robot and the pedestrians, Eq. 10, and the robot and the static obstacles, Eq. 11, is used by the robot to avoid possible collisions or unnecessary interactions with obstacles and other pedestrians (to see the exact equations of $\mathbf{f}_{r, o}^{\text {int }}$ and $\mathbf{f}_{r, j}^{i n t}$ the reader is referred to [3]).

\section{Social force model extension: moving goal}

As we have discussed previously, our goal it to make the couple human-robot capable of navigating side-by-side to meet the moving target person. We assume that the two people know each other and they will meet. Then, the robot adapts its behaviour to accompany one of these people to meet the other one. The best group geometrical configuration is side-by-side with a desired angle of $\pi / 2$ between the robot and the person being accompanied as is shown in Fig. 2 . Furthermore, the group's geometrical configuration has to be modified to avoid dynamic and static obstacles and the best path to intercept the target moving person must be computed. In this section, we explain the extension of the social force model to include a moving goal.

We also include an extension of the social force model to do the on-line adaptive side-by-side human-robot companion task, but the reader is referred 
to [17] for more details about the multiple on-line path computation to finally select the best group path.

To incorporate the moving goal, we have modified the social force model as is shown in Eq. 12, including a term to go to the target person $\beta \mathbf{f}_{r, t p}^{\text {goal }}\left(\mathcal{D}_{n}{ }^{t p}\right)$. Also, the moving goal has been taken into account to calculate all the possible paths. The part of $\alpha \mathbf{f}_{r, p}^{\text {goal }}\left(\mathcal{D}_{n}{ }^{p}\right)$ was modified in our previous work [17] to do an adaptive companion task and is explained in section 2. The $\alpha, \beta, \gamma$ and $\delta$ parameters are computed as is explained in our work [3]. Furthermore, in all the equations the super index of $p$ is referred to the person accompanied by the robot and the super index of $t p$ is referred to the person that the group wants to meet.

$$
\begin{gathered}
\mathbf{F}_{r}=\alpha \mathbf{f}_{r, t p}^{\text {goal }}\left(\mathcal{D}_{n}{ }^{t p}\right)+\beta \mathbf{f}_{r, p}^{\text {goal }}\left(\mathcal{D}_{n}{ }^{p}\right)+\left(\gamma \mathbf{F}_{r}^{p e r}+\delta \mathbf{F}_{r}{ }^{\text {obs }}\right) \\
1=\alpha+\beta+\gamma+\delta
\end{gathered}
$$

The attractive force assuming that the robot $r$ tries to adapt its velocity within a relaxation time $k^{-1}$, but taking into account at the same time that the final destination is a moving goal $\left(\mathcal{D}_{n}{ }^{t p}\right)$, which depends on the group direction of movement and the target person direction of movement, is computed as follows:

$$
\mathbf{f}_{r, t p}^{\text {goal }}\left(\mathcal{D}_{n}{ }^{t p}\right)=k\left(\mathbf{v}_{r}^{0}\left(\mathcal{D}_{n}{ }^{t p}\right)-\mathbf{v}_{r}\right)
$$

where $\mathbf{v}_{r}^{0}\left(\mathcal{D}_{n}{ }^{t p}\right)$ is the desired velocity vector to reach the goal according to the final moving destination $\mathcal{D}_{n}{ }^{t p}$ and $\mathbf{v}_{r}$ is the current velocity of the robot. This is the force that allows the robot to arrive to the final dynamic destination.

The $\mathcal{D}_{n}{ }^{t p}=\left(X_{d g}, Y_{d g}\right)$ is a point in the space and can be computed in two ways. First, if the group is not facing directly the direction of the target person, the robot has to compute the interception between the directions of the group's movement and the direction of the person. Second, if the group is in the same direction of the person, the group robot-human has to go to the predicted middle position between the group and the target person. Then the computation of these points is done as follows:

$$
\begin{gathered}
X_{d g}=\frac{Y_{t p}{ }^{t}-Y_{g}{ }^{t}+X_{g}{ }^{t} P_{g}-X_{t p}{ }^{t} P_{t p}}{P_{g}-P_{t p}} \\
Y_{d g}=P_{t p}\left(X_{d g}-X_{t p}{ }^{t}\right)+Y_{t p}{ }^{t} \\
P_{t p}=\frac{Y_{t p}{ }^{t+n}-Y_{t p}{ }^{t}}{X_{t p}{ }^{t+n}-X_{t p}{ }^{t}} \\
P_{g}=\frac{Y_{g}{ }^{t+n}-Y_{g}{ }^{t}}{X_{g}{ }^{t+n}-X_{g}{ }^{t}}
\end{gathered}
$$

where $\left(X_{t p}, Y_{t p}\right)^{t}$ is the actual position of the target person, $\left(X_{g}, Y_{g}\right)^{t}$ is the actual position of the group, $\left(X_{t p}, Y_{t p}\right)^{t+n}$ is the final predicted position of the person at the final of the window of time, $\left(X_{g}, Y_{g}\right)^{t+n}$ is final position of the group at the end of the window if they go in a straight line, $P_{t p}$ is the slope of the target person direction of movement and $P_{g}$ is the slope of the group direction of movement. We use a social force model to calculate the propagated 
positions of both, the group and the target person, inside our window of time. The initial an final positions were used to obtain the straight line of the direction of their movements.

\section{Simulation experiments}

In order to prove the good behaviour of our approach, we tested several situations where the group has to meet a companion person and avoid several dynamic and static obstacles at the same time. We have carried out more than 2400 simulations of the algorithm that combines the moving companion task of the group with the new part to meet the target person. We have used the same people simulator of [17], which uses the social force model [12] to obtain the people movement inside the environment. All the people desired velocities were randomly selected inside the interval of [0-1] $\mathrm{m} / \mathrm{s}$. Furthermore, we have modified our people simulator to include a more intelligent behaviour for the person who accompanies the robot. Now, the person behaviour approach resembles more the real people behaviour by planning several trajectories that avoids obstacles and other people. Then, the person can make turns and select among several possible paths when he/she needs to avoid dynamic or static obstacles. To obtain this behaviour, we have changed the behavior of this simulated person to use the anticipative kinodynamic planner [5]. Also, we have included the modification presented on that paper to allow the person to go towards the moving goal, which is used to meet the target person. Also, our simulated robot follows the laws of an non-honolomic vehicle and uses the on-line adaptive side-by-side human-robot companion of [17], in combination with the modification to include the moving goal.

We have used the proxemic distances described in [8]. Our algorithm uses a distance of $[1.5 \pm 0.5] \mathrm{m}$, between the robot and the person that accompanies. Furthermore, we have assumed that the two persons know each other, but not if the target person is familiar with robots. Due to that, in the simulation experiments we face the two persons with a minimum personal distance of $0.75 \mathrm{~m}$. Then, the robot moves in parallel with the accompanied person and faces the target person approaching him/her from one of his/her sides. Between the robot and the target person we have used a social distance of $1.75 \mathrm{~m}$ as minimum distance to approximate. We have selected this distance to prevent the robot to go too close to the person. In previous works, the robot's speed was around 0.4 $\mathrm{m} / \mathrm{s}$, but some people claimed that was too slow. Due to that, we have increased the person companion speed to $0.5 \mathrm{~m} / \mathrm{s}$. With respect to the robot speed, we allow up to $0.8 \mathrm{~m} / \mathrm{s}$ to permit changes of velocity to be positioned around the companion person. In general, the robot adapts its speed to the movement of the person being accompanied.

We have evaluated in all the cases the companion task and we have used the same metrics described in [17]. As we mentioned above, we consider the proxemic rules. Then, the robot makes a good performance if keeps its position on [1.25$2] \mathrm{m}$ with respect to the companion person, inside the social distances interval. For the companion angle, the best performance is when the robot only differs 


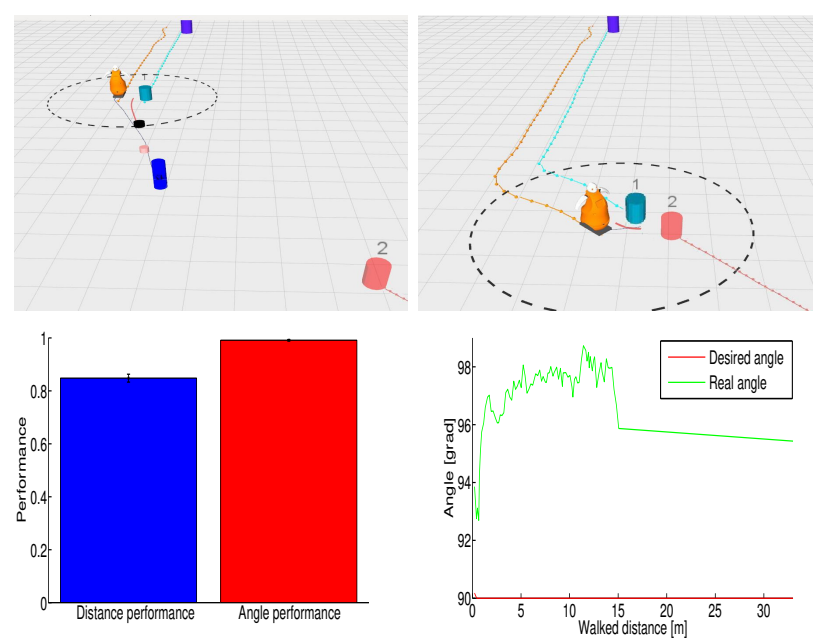

Fig. 3: The top plots show an intermediate state of the meeting task and the final state when the group and the target person where facing each other. The dark blue cylinder mark is the moving goal, the black dashed circle is the window of time to predict and calculate the paths, and the dashed lines are the all person and robot trajectories. At the bottom row, the plot of the left shows the results of the performance and the angle for all the simulations. The graph on the right shows the mean of the desired angle and the mean of the real angle for all the paths.

as much as plus-minus 10 degrees from the desired angle. Then, if this variation increases, we penalize each increment of 10 degrees with - 0.1 in performance.

For the meeting task to intercept the target person, we have considered also the proxemic rules [11]. Then, we consider a good performance when the simulated person ends near to the target person in a personal distance interval of [0.45-1.22] $\mathrm{m}$. Then, for the robot, we have considered a good performance if the approximation distance between it and the target person is inside the intervals of the person's social distances [1.22-3] $\mathrm{m}$. All the distances has been considered from the centre of person or robot. Furthermore, for the orientation angle, we have considered a good performance if the orientation of the group differs as much as 15 degrees of the contrary orientation of the target person, where the group is in the final position faced towards the target person.

The first group of simulations were carried out in an empty environment Fig. 3, where we tested only the new part of the moving goal. Furthermore in all the simulations, the target person starts from different positions to obtain different turns of the group: to the right, left and forward. In this case, we can see how the group modifies its formation to turn to the appropriate pose as expected, to meet the person, without any external changes due to dynamic or static obstacles. Regarding the results, the mean of performance in distance and angle were over the 0.8. The maximum difference between the desired and 


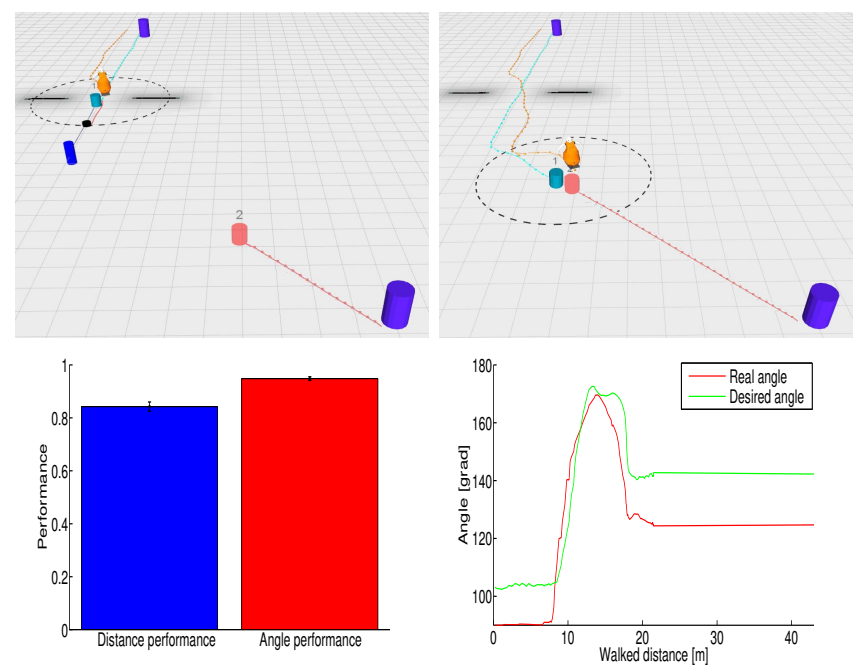

Fig. 4: The two top plots show an intermediate state of the meeting task, where the person and the robot pass, through the obstacle, and the final state when the group and the target are close. The plot on the bottom-left shows the results of the performance and the angle for all the simulations in the case that the target person meets the group from front-right side. In this case, the group has to avoid some obstacles of the environment. The plot on the right shows the mean of the desired angle of companion and the mean of the real angle for all the paths.

the real angle were 8 degrees. We also obtained very small oscillations in the side-by-side position.

In the second group of simulations, the environment has an static obstacle in the middle, which could be considered as a door, Fig. 4. Then, the algorithm has to solve the problems to go to the target person and the robot has to select the best path for the group and choose the best position around the person to avoid the obstacle in advance, in order to not collide and reduce the effort of the accompanied person. Regarding the results, the mean of performance in distance and angle were over the 0.8 . The maximum difference between the desired and the real angle was 24.96 degrees.

In the third group of simulations, the environment includes two groups of people that cross in the opposite direction of the group and forms a narrow dynamic corridor, Fig. 5. These simulated people have the same velocity which is randomly selected inside the interval of $[0-1] \mathrm{m} / \mathrm{s}$. In this case, we can see how the robot approaches the moving target while avoids other people. In addition, the robot has to predict the position of all people to behave appropriately. Regarding the results, the mean of performance in distance and angle were over the 0.9 . The maximum difference between the desired and the real angle was 13.29 degrees. 


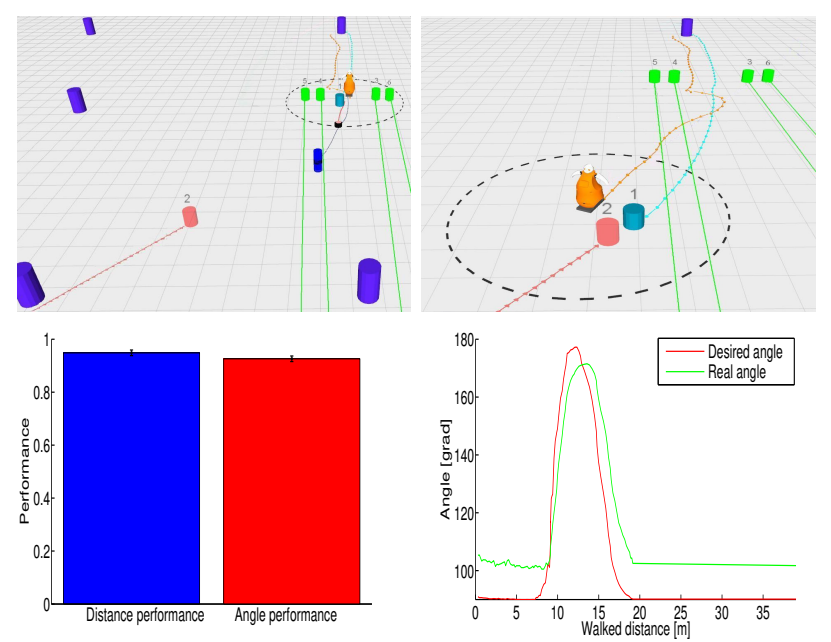

Fig. 5: The two top plots show an intermediate state of the meeting task and the final state when the group. The plot of the top-left shows the results of performance and angle for all the simulations of the case that the target person meet the group with the frontal direction. Here, the group has to avoid some groups of people that cross in the frontal direction. The plot of the right shows the mean of the desired angle of companion and the mean of the real angle of companion for all the paths.
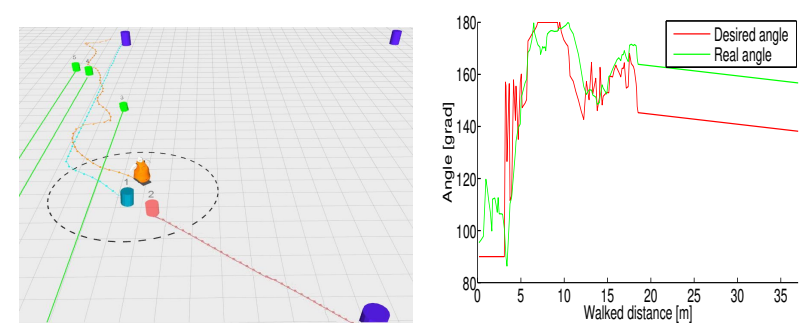

Fig. 6: The plot of the right show the final state of the meeting task when the group and the target person face each other at the closest distance. The plot of the left shows the change of the desired and real angles of the group while them walk avoiding the three persons until meet the target person.

The fourth group of simulations was similar to the third case, but now the people of the environment navigate in a independent way with different random velocities in the interval of $[0-1] \mathrm{m} / \mathrm{s}$, Fig. 6 . Then, the robot-human group has to deal with different situations where the group has to avoid one, two or three people before the group meets the target person as can be seen in the Fig. 6 . Regarding the results, the angle oscillations were less than 10 degrees in 3 meters. The maximum difference between the desired and the real angle was 20.18 degrees. 
The average of the walked distance for all the simulations was $38.59 \mathrm{~m}$, and the group performed it in a time average of $4.887 \mathrm{~min}$. To interpret the results in distance and time we have to take into account that the robot adapts its speed to the person being accompanied and in some cases their speeds decrease to avoid dynamic and static obstacles.

Regarding the results of all the simulations of the companion task, we obtained a distance average of 0.7 and an angle average of 0.8 , and their variances were 0.0239.Moreover, the mean of all the maximum difference between the desired and the real angle was 23.21 degrees. Therefore, we can conclude that with respect the companion task, our algorithm obtains good results in all the cases. With respect to meet the target person, the mean value, after all of the simulations of the final orientation between the group and the target person was 5.04 degrees, which is less than 15 degrees. And the mean value of the approximation distance between the robot and target person was $1.67 \mathrm{~m}$, which is inside the interval of the social distances. Finally, the mean value of the approximation distance between the companion person and target person was $1.04 \mathrm{~m}$, which is inside the interval of personal distances. These results show that our method also performs well on meeting the target person.

\section{Conclusions and future work}

In this paper, we have presented an adaptive human-robot side-by-side navigation approach, when a group of robot-human goes to meet a person that is moving. Our algorithm extends our past work described in [17]. The mayor contribution of this work is how we dynamically modify the intersection position to meet the target moving person, although there can be static or dynamic obstacles. To adapt the algorithm, we have extended the Social Force Model including the moving goal. The computation is done in real time. The new model has been tested on simulation and good results have been obtained, both in the accompaniment task and in the task of meeting a moving person.

Our future work will focus on adding the model in our robot and perform reallive experiments. Furthermore, in all cases of the simulation that we have made, we observed that the group goes directly facing the target person, but there can be situations were the group meets the target person just on the intersection. For this particular case, we will implement in the future a reorientation between the group and the target person to face directly each other.

\section{References}

1. Chen, Y., Wu, F., Shuai, W., Wang, N., Chen, R., Chen, X.: Kejia robot-an attractive shopping mall guider. In: International Conference on Social Robotics. pp. 145-154. Springer (2015)

2. Dautenhahn, K., Walters, M., Woods, S., Koay, K.L., Nehaniv, C.L., Sisbot, A., Alami, R., Siméon, T.: How may i serve you?: a robot companion approaching a seated person in a helping context. In: Proceedings of the 1st ACM SIGCHI/SIGART conference on Human-robot interaction. pp. 172-179. ACM (2006) 
3. Ferrer, G., Garrell, A., Herrero, F., Sanfeliu, A.: Robot social-aware navigation framework to accompany people walking side-by-side. Autonomous Robots pp. 1-19 (2016)

4. Ferrer, G., Garrell, A., Villamizar, M., Huerta, I., Sanfeliu, A.: Robot interactive learning through human assistance. (2013)

5. Ferrer, G., Sanfeliu, A.: Proactive kinodynamic planning using the extended social force model and human motion prediction in urban environments. In: IEEE/RSJ international conference on Intelligent robots and systems. pp. 1730-1735. IEEE (2014)

6. Ferrer, G., Sanfeliu, A.: Multi-objective cost-to-go functions on robot navigation in dynamic environments. In: IEEE/RSJ international conference on Intelligent robots and systems. pp. 3824-3829. IEEE (2015)

7. Garrell, A., Sanfeliu, A.: Cooperative social robots to accompany groups of people. The International Journal of Robotics Research 31(13), 1675-1701 (2012)

8. Garrell, A., Villamizar, M., Moreno-Noguer, F., Sanfeliu, A.: Teaching robots proactive behavior using human assistance. International Journal of Social Robotics 2(9), 231-249 (2017)

9. Graf, B., Wandosell, J.H., Schaeffer, C.: Flexible path planning for nonholonomic mobile robots. In: Proc. 4th European workshop on advanced Mobile Robots. pp. 199-206 (2001)

10. Haasch, A., Hohenner, S., Hüwel, S., Kleinehagenbrock, M., Lang, S., Toptsis, I., Fink, G.A., Fritsch, J., Wrede, B., Sagerer, G.: Biron-the bielefeld robot companion. In: Proc. Int. Workshop on Advances in Service Robotics. pp. 27-32. Citeseer (2004)

11. Hall, E.T., Edward, T.: Hall. the hidden dimension. Anchor Books New York 20, $71(1969)$

12. Helbing, D., Molnar, P.: Social force model for pedestrian dynamics. Physical review E 51(5), 4282 (1995)

13. Kuderer, M., Kretzschmar, H., Burgard, W.: Teaching mobile robots to cooperatively navigate in populated environments. In: IEEE/RSJ International Conference on Intelligent Robots and Systems. pp. 3138-3143. IEEE (2013)

14. Morales, Y., Kanda, T., Hagita, N.: Walking together: side by side walking model for an interacting robot. Journal of Human-Robot Interaction 3(2), 51-73 (2014)

15. Murakami, R., Morales Saiki, L.Y., Satake, S., Kanda, T., Ishiguro, H.: Destination unknown: walking side-by-side without knowing the goal. In: Proceedings of the ACM/IEEE international conference on Human-robot interaction. pp. 471-478. ACM (2014)

16. Prassler, E., Bank, D., Kluge, B., Hagele, M.: Key technologies in robot assistants: Motion coordination between a human and a mobile robot. Transactions on Control, Automation and Systems Engineering 4(1), 56-61 (2002)

17. Repiso, E., Ferrer, G., Sanfeliu, A.: On-line adaptive side-by-side human robot companion in dynamic urban environments. In: Intelligent robots and systems (IROS 2017), 2017 IEEE/RSJ international conference on. IEEE (2017)

18. Syrdal, D.S., Koay, K.L., Walters, M.L., Dautenhahn, K.: A personalized robot companion?-the role of individual differences on spatial preferences in hri scenarios. In: Robot and Human interactive Communication, 2007. RO-MAN 2007. The 16th IEEE International Symposium on. pp. 1143-1148. IEEE (2007) 